\title{
SHARPIN stabilizes $\beta$-catenin through a linear ubiquitination-independent manner to support gastric tumorigenesis
}

\author{
Liang Zhang ${ }^{1}$ - Qin Liu ${ }^{1} \cdot$ Ke-wei Liu ${ }^{1} \cdot$ Zhong-yi Qin $^{1}$ - Guang-xi Zhu ${ }^{1} \cdot$ Li-ting Shen $^{1} \cdot$ Ni Zhang ${ }^{1} \cdot$ Bi-ying Liu ${ }^{1}$. \\ Lin-rong Che ${ }^{1}$. Jin-yang $\mathrm{Li}^{1} \cdot$ Tao Wang $^{1} \cdot$ Liang-zhi Wen $^{1} \cdot$ Kai-jun Liu ${ }^{1} \cdot$ Yan Guo ${ }^{1} \cdot$ Xin-ru Yin ${ }^{1} \cdot$ Xing-wei Wang ${ }^{1}$. \\ Zhi-hua Zhou ${ }^{2} \cdot$ Hua-liang Xiao ${ }^{3}$. You-hong Cui ${ }^{4} \cdot$ Xiu-wu Bian ${ }^{4}$. Chun-hui Lan ${ }^{1} \cdot$ Dongfeng Chen $^{1} \cdot$ Bin Wang $^{1,4}$
}

Received: 8 July 2020 / Accepted: 19 October 2020 / Published online: 7 November 2020

(c) The International Gastric Cancer Association and The Japanese Gastric Cancer Association 2020

\begin{abstract}
Background Aberrant activation of Wnt/ $\beta$-catenin signaling by dysregulated post-translational protein modifications, especially ubiquitination is causally linked to cancer development and progression. Although Lys48-linked ubiquitination is known to regulate $\mathrm{Wnt} / \beta$-catenin signaling, it remains largely obscure how other types of ubiquitination, such as linear ubiquitination governs its signaling activity.

Methods The expression and regulatory mechanism of linear ubiquitin chain assembly complex (LUBAC) on Wnt/ $\beta$-catenin signaling was examined by immunoprecipitation, western blot and immunohistochemical staining. The ubiquitination status of $\beta$-catenin was detected by ubiquitination assay. The impacts of SHARPIN, a core component of LUBAC on malignant behaviors of gastric cancer cells were determined by various functional assays in vitro and in vivo.

Results Unlike a canonical role in promoting linear ubiquitination, SHARPIN specifically interacts with $\beta$-catenin to maintain its protein stability. Mechanistically, SHARPIN competes with the E3 ubiquitin ligase $\beta$-Trcp1 for $\beta$-catenin binding, thereby decreasing $\beta$-catenin ubiquitination levels to abolish its proteasomal degradation. Importantly, SHARPIN is required for invasiveness and malignant growth of gastric cancer cells in vitro and in vivo, a function that is largely dependent on its binding partner $\beta$-catenin. In line with these findings, elevated expression of SHARPIN in gastric cancer tissues is associated with disease malignancy and correlates with $\beta$-catenin expression levels.

Conclusions Our findings reveal a novel molecular link connecting linear ubiquitination machinery and Wnt/ $\beta$-catenin signaling via SHARPIN-mediated stabilization of $\beta$-catenin. Targeting the linear ubiquitination-independent function of SHARPIN could be exploited to inhibit the hyperactive $\beta$-catenin signaling in a subset of human gastric cancers.
\end{abstract}

Keywords Linear ubiquitination $\cdot$ SHARPIN $\cdot$ Wnt/ $\beta$-catenin signaling $\cdot$ Gastric cancer

Bin Wang, Dong-feng Chen and Chun-hui Lan contributed equally and are co-corresponding authors.

Liang Zhang and Qin Liu share co-first authorship.

Electronic supplementary material The online version of this article (https://doi.org/10.1007/s10120-020-01138-5) contains supplementary material, which is available to authorized users.

Chun-hui Lan

lanchunhui@tmmu.edu.cn

Dongfeng Chen

chendf1981@126.com

Bin Wang

wb_tmmu@126.com

Extended author information available on the last page of the article

\section{Introduction}

Protein homeostasis plays essential roles in many pathophysiological processes, including cell fate determination, and immune response, and cancer development [1]. As an important layer of regulatory machineries on protein homeostasis, the ubiquitin proteasome system (UPS) mainly controls the stability, subcellular localization or activity of target proteins. As such, ubiquitination, as one of the most extensively studied types of protein posttranslational modification, play a key role in modulating cell cycle progression and carcinogenesis [2]. The ubiquitination process is carried out by a cascade of reactions catalyzed by three sequential enzymes: ubiquitin-activating enzyme (E1), ubiquitin-carrier protein (E2), and ubiquitin-protein ligase (E3). The three-step 
catalytic cascade is activated by E1 in an ATP-dependent manner, and then activated ubiquitin is transferred to the cysteine residue on $\mathrm{E} 2$ before conjugation to a lysine residue on a target protein by E3 [3]. Multiple studies have shown the importance of ubiquitination in the development of cancer. Dysregulated ubiquitination of oncogenes or tumor suppressors is often found in tumorigenesis [4, 5]. For example, p53 is a well-known tumor suppressor and can be ubiquitinated by the E3 ligase MDM2 for proteasomal degradation [6]. Abnormal expression or mutations in MDM2 lead to p53 loss of function, thereby promoting tumorigenesis.

There are eight different polyubiquitin chains at present that are classified according to their inter-ubiquitin linkage. The seven lysine residues (Lys6, Lys11, Lys27, Lys29, Lys33, Lys48, and Lys63) in ubiquitin can form Lys-linked ubiquitin chains. The methionine (M1) in ubiquitin can function as an acceptor site to give rise to Met1-linked or 'linear' chains. Linear ubiquitination was catalyzed by linear ubiquitin chain assembly complex (LUBAC) composed of SHARPIN, HOIP and HOIL1, and hydrolyzed by linear ubiquitin-specific deubiquitinase (OTULIN). Different types of ubiquitin linkages determine distinct fate of the modified proteins. Lys48-linked ubiquitin chains are the predominant and best-studied linkage type. Their main function is to mediate the degradation of target proteins and regulate protein stability through the UPS [7]. Lys11-linked and Lys29-linked chains have been found to act as additional proteasomal degradation signals [8]. In contrast, Lys63linked and linearly linked chains serve as specific binding sites for nonproteolytic signaling. Lys63-linked chains have been well studied in the regulation of DNA damage, protein trafficking and the inflammatory response, especially in tumorigenesis. For instance, Lys63-linked chains serve as a platform for the recruitment TGF- $\beta$ activating kinase 1 (TAK1) and activation of IKB (IKK) kinase complexes, thereby resulting in NF- $\mathrm{\kappa B}$ activation [9]. Linear ubiquitination is involved in immunity, lymphocyte development and chronic inflammation [10]. Recent studies have indicated that linear ubiquitination may also play a role in the pathogenesis of tumors [11, 12]. However, the exact functions and underlying mechanisms of linear ubiquitination machinery in human cancers are yet to be determined.

Increasing evidence indicates that $\mathrm{Wnt} / \beta$-catenin signaling is closely involved in the development of human malignancies including gastric cancer (GC). Aberrant activation of Wnt signaling is detected in more than 50\% of GC [13]. In the absence of Wnt ligand stimulation (inactive state), $\beta$-catenin is phosphorylated by a cytoplasmic multisubunit destruction complex and then degraded by ubiquitination. When Wnt ligands bind to the corresponding Frizzled/ low-density lipoprotein receptor-related protein (LRP) coreceptor, a signaling cascade is initiated, and the ubiquitination of $\beta$-catenin by $\beta$-TrcpE3 ligase is inhibited, which consequently results in the accumulation of $\beta$-catenin in the cytoplasm and then translocation into the nucleus. Through binding to $\mathrm{T}$ cell factor/lymphoid enhancer factor (TCF/ LEF) transcription factors, $\beta$-catenin initiates the transcription of multiple target genes, which regulates cell proliferation, differentiation, migration and tumorigenesis [14]. In the Wnt signaling cascade, ubiquitination modification plays an important regulatory role. It is noteworthy that linear ubiquitination is also implicated in the regulation of Wnt signaling in a recent study [15]. OTULIN mutation causes embryonic angiogenic deficits as a result of dysregulation of Wnt signaling. Although OTULIN was found to interact with dishevelled-2 (DVL2) to regulate Wnt signaling, the underlying mechanisms are not clear. Whether and how LUBAC components regulate Wnt signaling to impact on tumorigenesis warrants further studies.

In the present study, we have identified that SHARPIN, a key component of LUBAC, specifically interacts with, and inhibits ubiquitination and protesomal degradation of $\beta$-catenin in a linear ubiquitination-independent manner. In human GC cells, SHARPIN enhances $\beta$-catenin signaling activity to promote malignant growth and progression. Therefore, targeting the SHARPIN- $\beta$-catenin axis could be a new potential strategy for the treatment of human GC.

\section{Materials and methods}

\section{Tissue samples from GC patients}

One hundred eighty paraffin-embedded samples were obtained from patients diagnosed with GC who underwent gastrectomy in Southwest Hospital between January 2006 and December 2007. The samples contained GC tissues and adjacent mucosa. Clinical features of samples were obtained by querying the results of relevant examinations of GC patients. Follow-up studies were performed, and GC recurrence or patient death was recorded. All patients provided written informed consent. The procedure was approved by the Institutional Research Ethics Committee of Southwest Hospital and conducted according Declaration of Helsinki Principles.

\section{Reagents and antibodies}

Rabbit polyclonal anti-SHARPIN antibody, anti-C-MYC antibody, anti-CD44 antibody, anti- $\beta$-catenin and mouse monoclonal anti-GAPDH antibody were purchased from Proteintech. Anti- $\beta$-catenin antibody and anti- $\beta$-Trcp 1 antibody were obtained from Cell Signaling Technology (CST). Rabbit monoclonal and mouse monoclonal anti-HA tag, anti-Myc tag and anti-strep tag antibodies were from MBL. Anti-linear ubiquitin antibody (clone LUB9) was purchased 
from Merck Millipore. Normal mouse and rabbit anti-IgG antibodies were obtained from Beyotime. The detailed information of the antibodies was shown in Supplementary Table S1. Chemical reagents MG132 and puromycin were purchased from Beyotime. Bafilomycin A1 (lysosome inhibitor) was purchased from Selleckchem. Cycloheximide was obtained from APExBIO, and blasticidin was purchased from Solarbio.

\section{Cell culture}

The human embryonic kidney cell line HEK-293T and the GC cell line HGC-27 were purchased from the Cell Bank of Shanghai Institute of Cell Biology (Shanghai, China). The primary XN0422 GC cell line was obtained from the Institute of Pathology and Southwest Cancer Center, Southwest Hospital, as described previously [16]. HEK-293T cells were cultured in DMEM (HyClone, USA), while XN0422 and HGC-27 cells were cultured in RPMI-1640 (HyClone, USA). All cell lines were maintained in medium supplemented with $10 \%$ fetal bovine serum (Thermo Scientific, USA), 100 units $/ \mathrm{ml}$ penicillin, and $100 \mu \mathrm{g} / \mathrm{ml}$ streptomycin at $37{ }^{\circ} \mathrm{C}$ in a $5 \% \mathrm{CO} 2$ humidified incubator.

\section{Plasmids, RNA interference and lentivirus production}

Plasmids for Myc-HOIP, Myc-HOIL1, Myc-SHARPIN, Myc-OTULIN, HA-DVL2, HA- $\beta$-catenin and His-ub were purchased from Addgene. The SA mutant $\beta$-catenin plasmid, in which four serine/threonine residues (S33, S37, T41 and S45) were replaced by alanine, was constructed by point mutation methods. The internally tagged Ub (INT-Ub) for the study of linear ubiquitination was kindly gifted by Professor Koraljka Husnjak (Goethe University Frankfurt Medical Faculty) [17]. To knockdown SHARPIN, three siRNAs were constructed by Sangon Biotech in Shanghai (China). A scramble siRNA was used as a negative control. Plasmid and siRNA transfection were performed using Lipofectamine ${ }^{\mathrm{TM}}$ 3000 Transfection Reagent (Invitrogen ${ }^{\mathrm{TM}}$ ).

The oligonucleotide sequences targeting SHARPIN were as follows: siRNA\#1: 5'-CCUGAGGCAGAUCUU CCUATT-3'; siRNA\#2: 5'-CCCUGAGUGUUCAGCUUC ATT-3'; and siRNA\#3: 5'-GCUGCAGGUCACACUUGA ATT-3'. Western blot analysis was performed to assess the efficacy of each siRNA. The siRNA with the most effective knockdown efficacy was selected for further experiments. Lentiviruses for SHARPIN interference and $\beta$-catenin overexpression were constructed as previously described [13]. For stable knockdown of SHARPIN, XN0422 cells infected with interference lentivirus were subjected to puromycin selection for 5 days. Then, for rescue experiments, SHARPIN knockdown-XN0422 cells were infected with $\beta$-catenin overexpression (OE) lentivirus and subjected to by blasticidin selection for 7 days.

\section{Ubiquitination assay}

The ubiquitination assay was performed using a nickelnitrilotriacetic acid (Ni-NTA) pull-down assay as previously described [18]. Briefly, after transfection with the indicated plasmids in $293 \mathrm{~T}$ cells, the cellular proteins were harvested using Buffer A (6 M guanidine-HCl, 0.1 M $\mathrm{Na}_{2} \mathrm{HPO} 4 / \mathrm{NaH}_{2} \mathrm{PO} 4,10 \mathrm{mM}$ imidazole ( $\mathrm{pH}$ 8.0) for $250 \mathrm{ml}$ ) after pretreatment with MG132 for $8 \mathrm{~h}$. Then, cell lysates were sonicated for $35 \mathrm{~s}$ at amplitude $35 \%$ and centrifuged for $10 \mathrm{~min}$ at $4{ }^{\circ} \mathrm{C}$. Supernatant lysates were incubated with prewashed Ni-NTA (QIAGEN) for $3 \mathrm{~h}$ at room temperature. Then, lysates were subjected to three washing steps in Buffer A, Buffer A/Buffer TI (1:3) and Buffer TI (25 mM Tris-CL, $20 \mathrm{mM}$ imidazole). Finally, the pulled-down proteins were eluted by boiling in SDS-PAGE (2X) buffer and prepared for western blot analysis.

\section{Protein half-life assay}

Cycloheximide (CHX) pulse-chase experiments were performed to detect the protein half-life as previously described [18]. After treatment with the indicated treatments, the cells were evenly divided into six-well plates. According to the designed time point, $\mathrm{CHX}$ was added to the cell culture medium. Then, cellular proteins were collected and prepared for western blot analysis.

\section{Immunohistochemical (IHC) staining}

IHC was performed using Histostain ${ }^{\mathrm{TM}}$-SP Kits (ZSGB$\mathrm{BIO}$, China) according to the manufacturer's protocol. In brief, the tissue sections were deparaffinized and hydrated in graded ethanol. After washing with PBS, antigen retrieval was performed with citrate repair solution by heating in a microwave oven. Endogenous peroxidase activity was blocked, and sections were blocked with goat serum. Then, the sections were incubated with the corresponding primary antibody overnight at $4{ }^{\circ} \mathrm{C}$. The next day, horseradish peroxidase (HRP)-conjugated secondary antibody was added, and the immunohistochemical reaction was detected by a DAB kit (ZSGB-BIO, China). Finally, the sections were stained with hematoxylin, dehydrated and mounted with neutral resin. IHC staining scores in GC tissues were assessed by two pathologists following a previously described protocol [14]. For quantification of $\beta$-catenin expression, we mainly analyzed its nuclear staining intensity. The IHC score was the product of the strength score and the distribution score. The staining strength score was classified as negative: 0 , weak: 1 , moderate: 2 or strong: 3 . The distribution score 
was classified as negative: 0 , positive staining area $\leq 25 \%$ : $1,26 \%-50 \%: 2,51 \%-75 \%: 3, \geq 76 \%: 4$. A score $\geq 6$ was designated as high expression. A score $<6$ was classified as low expression. Five representative staining fields of each tumor sample were analyzed.

\section{Colony formation assay}

To estimate the growth capacity of GC cells, a colony formation assay was performed as previously described [19]. Approximately 500-1000 GC cells were seeded in six-well plates containing 10\% FBS RPMI-1640 medium. After 10-15 days, visible colonies were washed with PBS buffer three times and fixed with $4 \%$ paraformaldehyde for $25 \mathrm{~min}$. Then, colonies were stained with crystal violet for $10 \mathrm{~min}$ and washed with distilled water three times. Finally, the colonies were quantified and analyzed.

\section{Soft agar colony formation assay}

A soft agar colony formation assay was performed to detect the anchorage-independent growth capacity of GC cells, as described previously [19]. In brief, $2 \mathrm{ml}$ of medium containing $1 \%$ agar was added to a 6 -well plate as the bottom layer. After the bottom layers were solidified, 1000 GC cells suspended in $0.5 \%$ agar RPMI-1640 medium were seeded into the top layer. Then, after 20-30 days, visible colonies were stained with iodonitrotetrazolium chloride (Sigma), quantified and analyzed.

\section{Mouse xenograft tumor model}

All animal experiments were approved by the Animal Research Ethics Committee of Third Military Medical University (Army Medical University) and complied with the Guidelines for Animal Experiments of Laboratory Animals. XN0422/scramble, XN0422/sh-SHARPIN, shSHARPIN XN0422/vector and sh-SHARPIN XN0422/ OE- $\beta$-catenin cells were constructed. Approximately $1 \times 10^{6}$ cells were injected subcutaneously into the nude mice. After 5 weeks, the nude mice were sacrificed humanely. Tumor weight and volume were measured. Volume of tumor $=$ length $\times$ width $^{2} \times 0.5$. The tumor tissues were removed and sliced into paraffin sections for IHC analysis.

\section{Statistical analysis}

All of the experiments were repeated for at least three times independently. The quantitative data are presented as the mean \pm SD of biological replicates. The results were analyzed by independent sample $t$ test or the Mann-Whitney $U$ test for continuous variables and by Chi-square analysis or Fisher's exact test for categorical variables. Cox proportional-hazards model was established for univariate and multivariate analyses. Statistical analyses were performed using IBM SPSS Statistics for Windows, Version 22.0 (IBM Corp., Armonk, NY). Spearman's analysis of correlation analyses and Kaplan-Meier analysis of survival analysis were analyzed using GraphPad Prism 6 (GraphPad, USA). Values of $P<0.05$ were considered statistically significant.

\section{Results}

\section{SHARPIN promotes $\beta$-catenin expression and enhances its signaling activity in a linear ubiquitination-independent manner}

To dissect the regulatory mechanisms of linear ubiquitination pathway on Wnt/ $\beta$-catenin signaling, HEK-293T cells were transfected with LUBAC component, or OTULIN constructs for western blot analysis. Consistent with the results of previous studies [15], ectopic express of OTULIN increased $\beta$-catenin levels (Fig. 1a). Importantly, we found that SHARPIN also increased $\beta$-catenin expression, while HOIP and HOIL1 inhibited $\beta$-catenin expression (Fig. 1a). Considering that the LUBAC-OTULIN axis might modulate $\beta$-catenin activity by (de)ubiquitination, we sought to investigate the interaction between the LUBAC-OTULIN axis and $\beta$-catenin. Immunoprecipitation results showed that SHARPIN, but not HOIP, HOIL1 and OTULIN, specifically interacted with $\beta$-catenin (Fig. 1b). Moreover, an endogenous interaction between SHARPIN and $\beta$-catenin was also confirmed in human GC cells (Fig. 1c).

To further demonstrate the effect of SHARPIN on $\beta$-catenin activity, ectopic expression of SHARPIN increased the protein levels of $\beta$-catenin and its target gene $\mathrm{C}$-myc in a dose-dependent manner (Fig. 1d). Moreover, depletion of endogenous SHARPIN in CTNNB1-wild type XN0422 and HGC-27 GC cells downregulated the levels of $\beta$-catenin as well as its downstream targets C-myc and CD44 in GC cells (Fig. 1e and S1a, Supplementary Table S2).

We next characterize how SHARPIN may regulate $\beta$-catenin activity. NF- $\kappa \mathrm{B}$-essential modulator (NEMO), a known substrate of linear ubiquitination [10], was used as a positive control in our experimental system (Fig. 1f, left panel). The immunoprecipitation results demonstrated that $\beta$-catenin was not linearly ubiquitinated by LUBAC (Fig. 1f, right panel). Additionally, dishevelled-2 (DVL2) which was known to interact with OTULIN, was also not modified by linear ubiquitination (Fig. S1b). $\mathrm{Wnt} / \beta$-catenin signaling pathway is frequently activated by mutation of APC and $\beta$-catenin, or promoter methylation of Wnt antagonists, such as SFRP1, SFRP2, SFRP5, DKK2 and DKK3 in human cancers. However, 
a

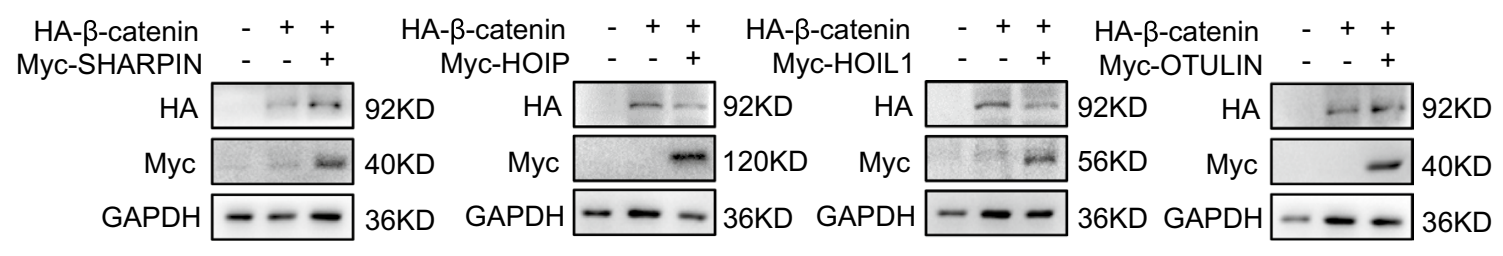

b

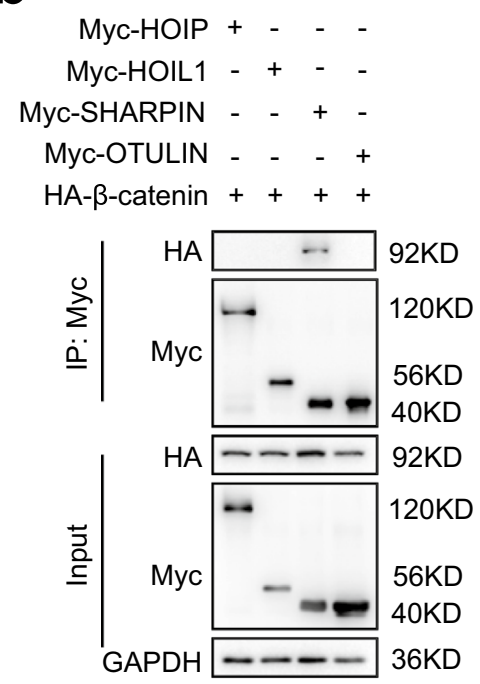

e

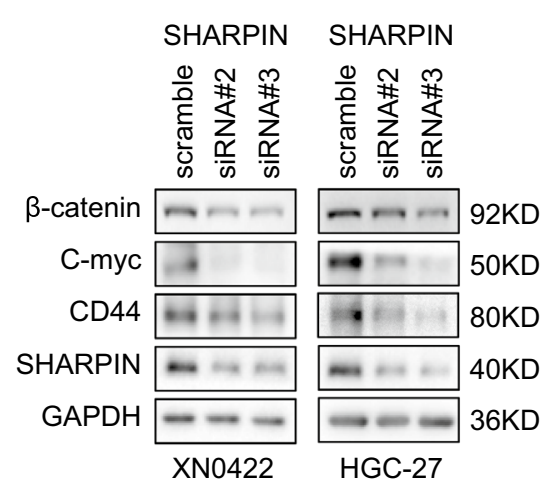

C

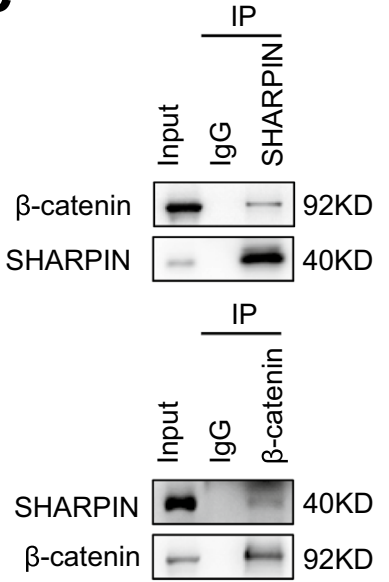

d

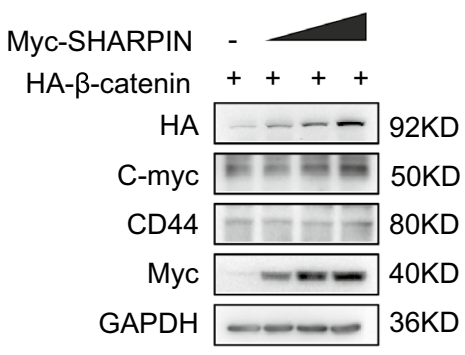

f
INT-Ub ++

HA-NEMO ++

Myc-HOIP - +

Myc-SHARPIN - +

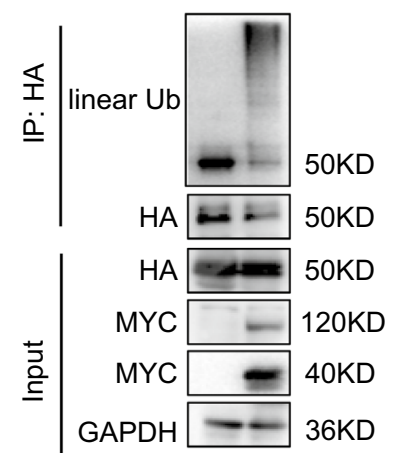

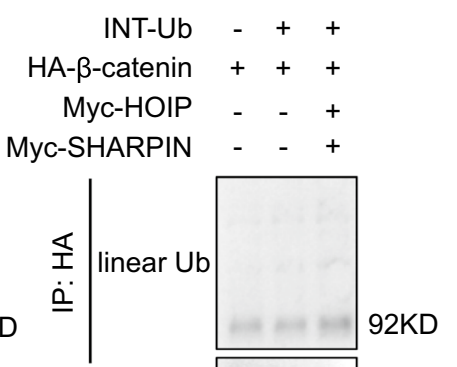

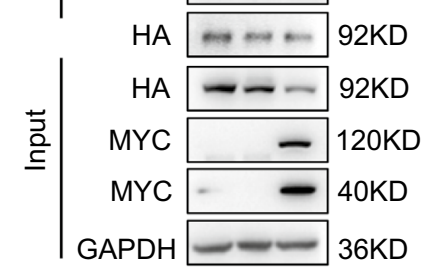

Fig. 1 SHARPIN specifically promotes $\beta$-catenin expression and its activity in a linear ubiquitination-independent manner. a HEK-293T cells transfected with the indicated constructs. After $48 \mathrm{~h}$ of transfection, cell lysates were collected for immunoblot analysis to detect the level of HA-tagged $\beta$-catenin. b HEK-293 T cells were transfected with the indicated constructs. Then, anti-Myc immunoprecipitates were collected for immunoblot analysis with the indicated antibodies to detect the interaction between $\beta$-catenin and HOIP, HOIL1, SHARPIN and OTULIN. c Immunoprecipitation was performed in HGC-27 cells to detect endogenous interactions between SHARPIN and $\beta$-catenin. IgG was used as a negative control. d HEK-293T cells were transfected with the indicated vectors. Immunoblot analysis was performed to detect the expression of HA-tagged $\beta$-catenin, C-myc and CD44 in HEK-293T cells transfected with increasing amounts of SHARPIN. e SHARPIN was knocked down by the indicated siRNA in XN0422 and HGC-27 cells. Then, immunoblot analysis was performed to detect the expression of $\beta$-catenin, $\mathrm{C}$-myc and CD44. $\mathbf{f}$ HEK-293T cells transfected with the indicated constructs. INT-Ub expression was induced by doxycycline $(1 \mu \mathrm{g} / \mathrm{ml} ; 24 \mathrm{~h})$ followed by MG132 treatment $(20 \mu \mathrm{mol}$ for $8 \mathrm{~h})$. Whole cell lysis, anti-HA immunoprecipitates were collected for immunoblot analysis with the indicated antibodies. Left panel, NEMO as a positive control for linear ubiquitination assay 
a

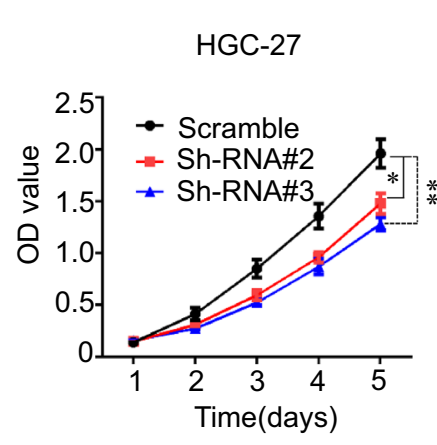

b

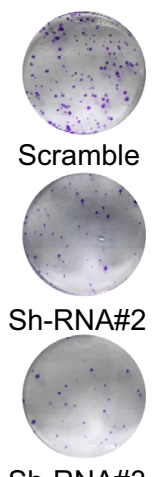

d

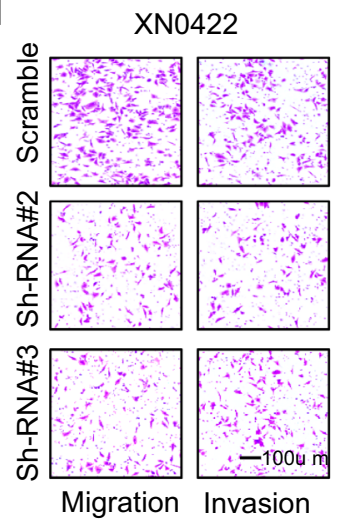

f

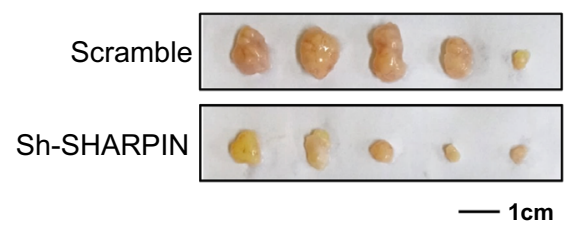

i

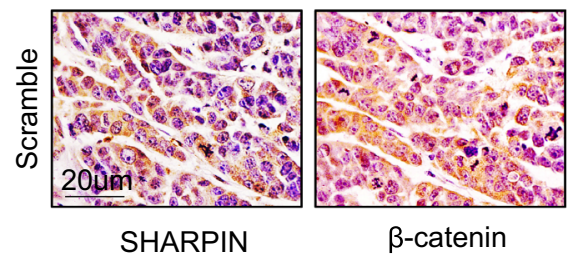

by analyzing TCGA gastric cancer dataset, we found no significant difference between SHARPIN expression and the mutation status of $\beta$-catenin and APC (Fig. S1c, d). Moreover, SHARPIN expression is largely not associated with methylation status of the Wnt antagonists

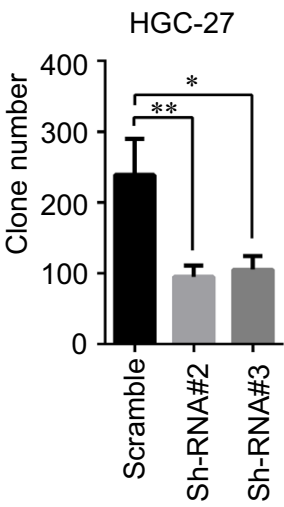

C

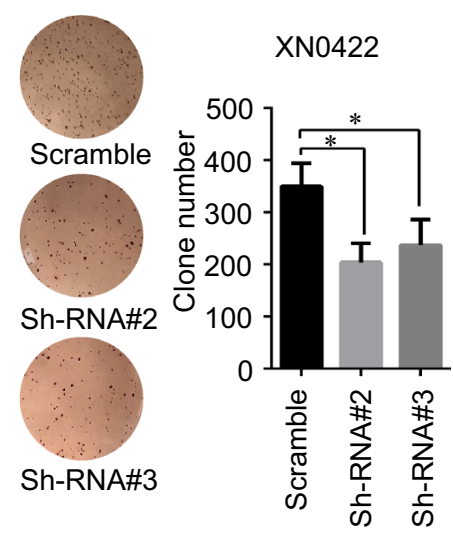

e

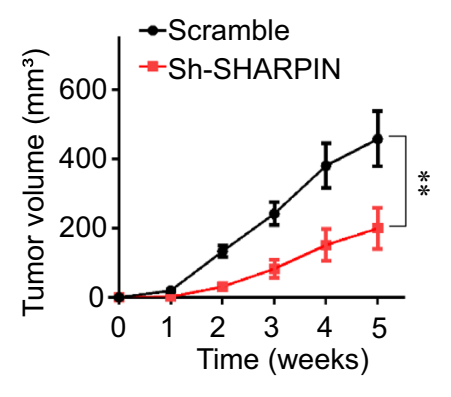

h
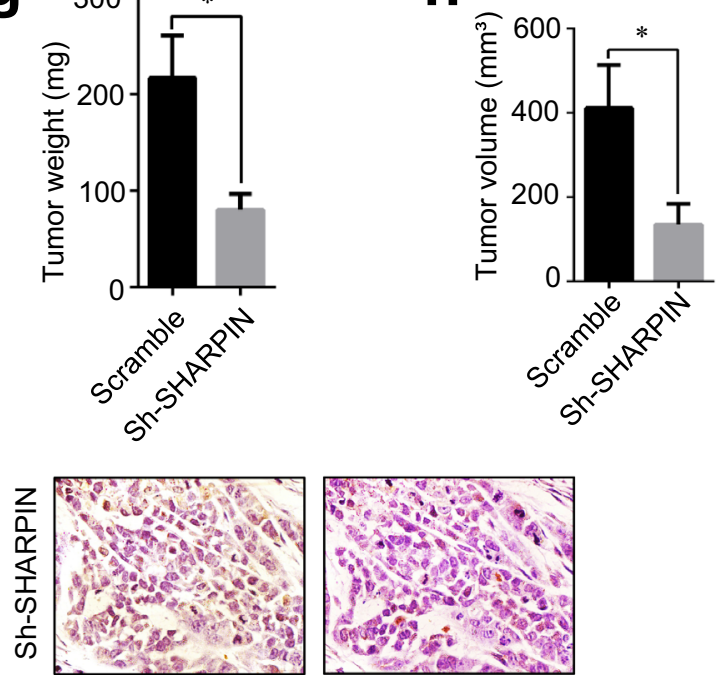

SHARPIN

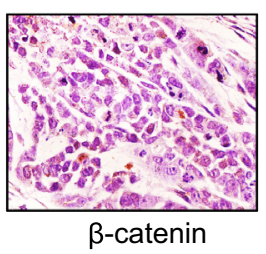

(Fig. S1e-i). Together, these data reveal a previously unknown role of the LUBAC component SHARPIN in stabilizing $\beta$-catenin to promote its signaling activity, which is unlikely through a linear ubiquitination regulatory mechanism. 
4Fig. 2 SHARPIN sustains malignant growth of GC cells and its $\beta$-catenin signaling. a CCK8 analysis of the proliferation of HGC-27 cells with SHARPIN knockdown by SHARPIN shRNA or control shRNA. b Clone formation assay was performed to detect the proliferation of HGC-27 cells with SHARPIN knockdown by SHARPIN shRNA or control shRNA. c Soft agar clone formation assay was performed to detect the proliferation of XN0422 cells with SHARPIN knockdown by SHARPIN shRNA or control shRNA. d Transwell migration and invasion assays were performed to detect the migration and invasion ability of XN0422 cells transfected with SHARPIN shRNA or control shRNA. Quantitative data are presented as the mean \pm SD. $* P<0.05$; $* * * P<0.001$. e XN0422 scramble and shSHARPIN cells were subcutaneously injected into the hind limbs of nude mice ( $n=5 /$ group, $1 \times 10^{6}$ cells per injection). Xenograft tumors were dissected and photographed. f Growth curve analysis of tumor proliferation activity after treatment with scramble or sh-SHARPIN. $\mathbf{g}-\mathbf{h}$. The xenograft tumors were dissected, and tumor weight and volume were measured in each group $(P<0.05)$. Data are presented as the mean \pm SD. i Representative histopathology images of IHC staining of SHARPIN and $\beta$-catenin in tumor sections

\section{Depletion of SHARPIN inhibits malignant growth and invasiveness of GC cells and reduces $\beta$-catenin activity.}

Given an established oncogenic function of $\beta$-catenin in various human cancers, we propose that SHARPIN, as a positive regulator of $\beta$-catenin, may also regulate malignant phenotypes of cancer cells. To this end, endogenous SHARPIN was depleted in XN0422 and HGC-27 GC cells using lentiviral vectors carrying two independent shRNAs (Sh-RNA \#2 and \#3). The downregulation of SHARPIN inhibited GC cell proliferation and colony formation (Figs. 2a, b, S2a, b), leading to decreased anchorage-independent growth capacity in soft agar (Figs. 2c and S2c). In addition, downregulation of SHARPIN inhibited GC cell migration and invasion in vitro (Figs. $2 \mathrm{~d}$ and S2d).

Next, we examined whether endogenous SHARPIN promotes growth of xenograft tumors in vivo. Compared with the scramble control mice, knockdown of SHARPIN markedly inhibited tumor growth (Fig. 2e, f), resulting in smaller tumor weight and volume in nude mice (Fig. 2g, h). Further analysis of the dissected tumors revealed that loss of SHARPIN was associated reduced $\beta$-catenin levels (Fig. 2i). Consistently, SHARPIN knockdown inhibited the expression of $\beta$-catenin target genes, such as c-myc and CD44 (Fig. S2e), suggesting decreased $\beta$-catenin signaling activity in this scenario. Collectively, these data demonstrated that SHARPIN was essential for maintaining $\beta$-catenin signaling activity and malignant growth of GC cells.
SHARPIN decreases $\beta$-catenin ubiquitination levels in part by competing with the E3 ligase $\beta$-Trcp1 for $\beta$-catenin binding

We next sought to understand mechanistically how SHARPIN regulates the expression of $\beta$-catenin. Through analyzing the TCGA database [20], SHARPIN was not correlated with mRNA level of $\beta$-catenin (gene symbol: CTNNB1, Fig. S3a, left panel). Consistently, ectopic expression of SHARPIN minimally affected $\beta$-catenin mRNA levels (Fig. S3b). However, SHARPIN levels was significantly correlated with the mRNA level of a putative $\beta$-catenin target gene myc (Fig. S3a, right panel). These results, together with a previous study [21], indicate that SHARPIN may regulate $\beta$-catenin expression and activity at posttranscriptional levels.

Consistent with our hypothesis, decreased $\beta$-catenin expression was rescued by the proteasome inhibitor MG132 in SHARPIN-depleted cells (Fig. 3a). Moreover, ectopic expression of SHARPIN prolonged, while depleting endogenous SHARPIN shortened the half-life of $\beta$-catenin protein (Figs. 3b and S3c). Moreover, ectopic expression of SHARPIN suppressed polyubiquitination status of $\beta$-catenin (Figs. $3 \mathrm{c}$ and S3d). Thus, SHARPIN reduced the ubiquitination levels of $\beta$-catenin, thereby suppressing its proteasomal degradation to maintain protein stability.

Beta-catenin was ubiquitinated by several E3 ubiquitin ligases especially, $\beta$-Trcp 1 , for degradation by UPS [22]. We examined whether SHARPIN influenced the recognition of $\beta$-catenin by E3 ubiquitin ligase, thus attenuating its ubiquitination levels. Ectopic expression of SHARPIN decreased the levels of $\beta$-catenin-binding $\beta$-Trcp1 in a dosedependent manner (Fig. 3d). Conversely, overexpression of $\beta$-Trcp1 also downregulated the level of $\beta$-catenin-binding SHARPIN (Fig. 3e). Furthermore, knockdown of SHARPIN enhanced the interaction between $\beta$-catenin and $\beta$-Trcp 1 at endogenous levels (Fig. 3f). Thus, SHARPIN might compete with $\beta$-Trcp 1 for binding $\beta$-catenin, thereby suppressing the ubiquitination of $\beta$-catenin. In line with our speculation, overexpression of SHARPIN partially reduced the ubiquitination, and consequently degradation mediated by $\beta$-Trcp 1 in cells (Fig. $3 g, h$ ). These results suggest that SHARPIN decreased $\beta$-catenin ubiquitination in part through competing with $\beta$-Trcp 1 for $\beta$-catenin binding.

\section{SHARPIN competes with $\beta$-Trcp1 for $\beta$-catenin binding partly by occupying similar residues of $\beta$-catenin}

To further investigate the underlying competitive mechanism between SHARPIN and $\beta$-Trcp1 for $\beta$-catenin binding, we established SA mutant $\beta$-catenin, in which four serine/ threonine residues (S33, S37, T41 and S45) were replaced 

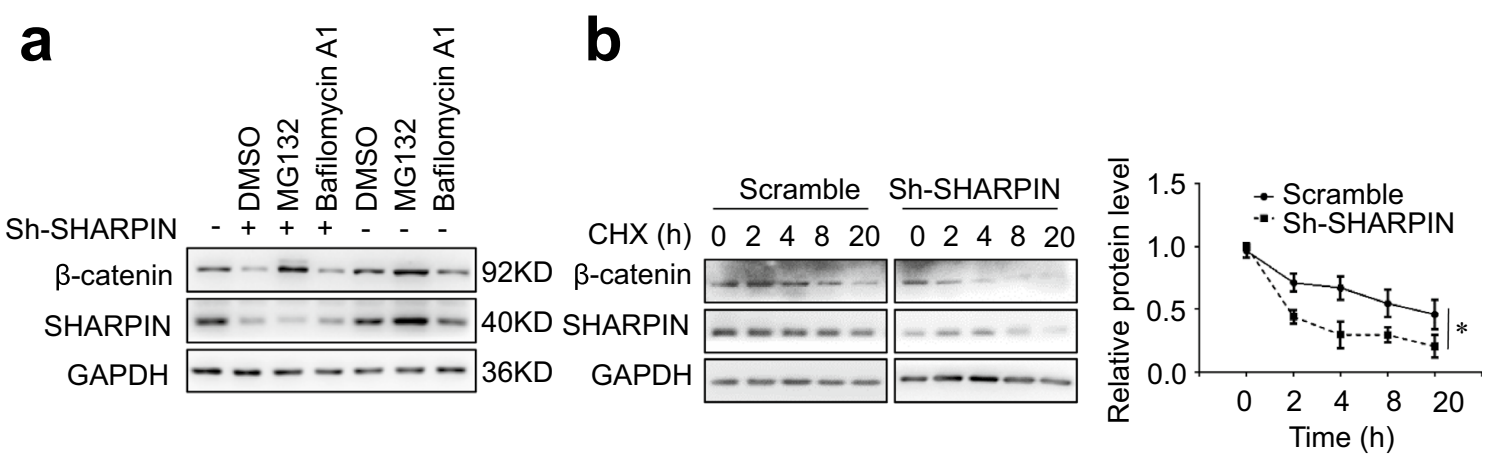

C

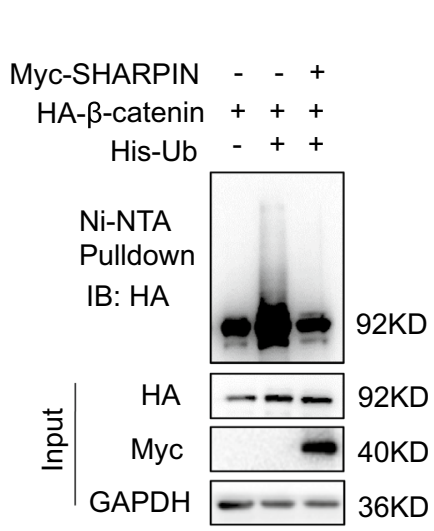

f

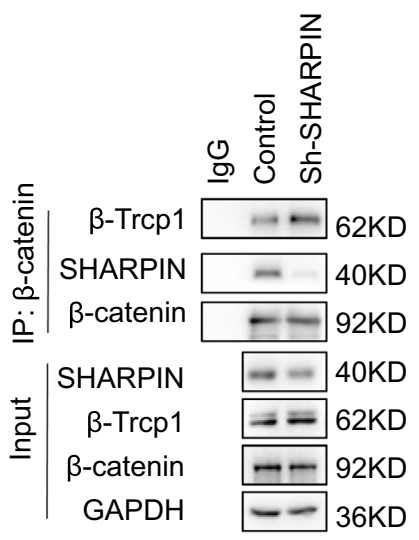

d

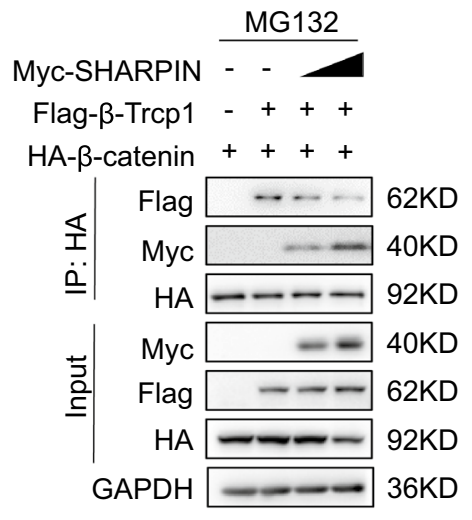

g

His-Ub - +++

Flag- $\beta-\operatorname{Trcp} 1-\quad++$

Myc-SHARPIN - - -+

HA- $\beta$-catenin ++++

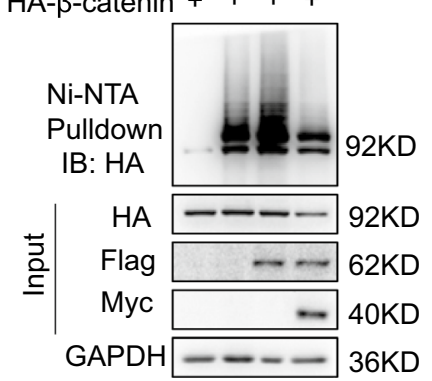

e

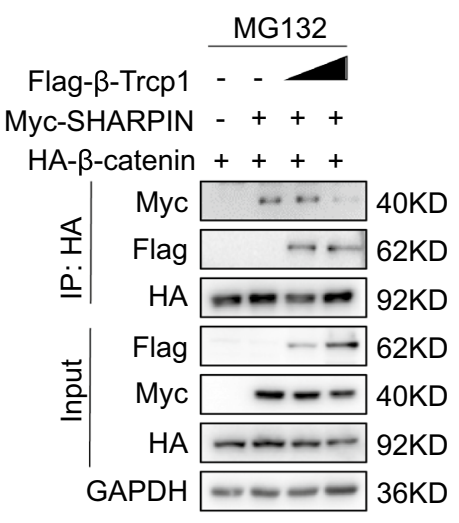

h

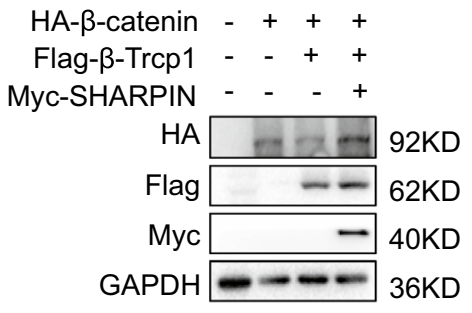

Fig. 3 SHARPIN competes with the E3 ligase $\beta$-Trcp1 for $\beta$-catenin binding, thereby reducing $\beta$-catenin ubiquitination levels. a XN0422 cells were transfected with scramble or sh-SHARPIN RNA. Then, the cells were treated with DMSO, MG132 or bafilomycin A1. Cell lysates were collected for immunoblot analysis. b XN0422/scramble and XN0422/shSHARPIN cells were treated with cycloheximide (CHX) for the indicated time course. Endogenous $\beta$-catenin was detected by immunoblot analysis (left). Quantification of the $\beta$-catenin/GAPDH ratio is shown in the right panel. c HEK-293 T cells transfected with the indicated constructs followed by MG132 treatment $(20 \mu \mathrm{mol}$ for $8 \mathrm{~h})$. Ni-NTA pull-downs were performed for immunoblot analysis. d-e. SHARPIN competed with $\beta$-Trcp1 for binding $\beta$-catenin. HEK-293T cells were transfected with MycSHARPIN, Flag- $\beta$-Trcp1 and HA- $\beta$-catenin plasmids and then incu- bated with MG132 for $8 \mathrm{~h}$. Anti-HA immunoprecipitates were collected for immunoblot analysis with the indicated antibodies. $f$. SHARPIN competed with $\beta$-Trcp1 for binding $\beta$-catenin at endogenous levels. XN0422/control and XN0422/shSHARPIN cells were treated with MG132 for $8 \mathrm{~h}$. Anti- $\beta$-catenin immunoprecipitates were collected for immunoblot analysis. g SHARPIN inhibited the $\beta$-Trcp1-mediated ubiquitination of $\beta$-catenin in a competitive manner. HEK-293 T cells were transfected with the indicated plasmids and incubated with MG132 for $8 \mathrm{~h}$. Ni-NTA pull-downs were performed for immunoblot analysis. h. SHARPIN antagonized $\beta$-Trcp1mediated degradation of $\beta$-catenin. HEK-293T cells transfected with the indicated plasmids and lysed were collected for immunoblot analysis 
by alanine (A) to abolish $\beta$-catenin phosphorylation and recognition by $\beta$-Trcp1 [23]. Compared to wild-type $\beta$-catenin, the mutant $\beta$-catenin that did not associate with $\beta$-Trcp1 displayed enhanced binding affinity with SHARPIN, (Fig. 4a, b). Moreover, overexpression of SHARPIN increased the wild-type $\beta$-catenin level but not mutant $\beta$-catenin level (Fig. 4c). Consistently, SHARPIN decreased the ubiquitination level of wild-type $\beta$-catenin, but not mutant $\beta$-catenin, therefore, could not extend the half-life of mutant $\beta$-catenin (Fig. 4d, e). These results further indicate that SHARPIN and $\beta$-Trcp1 might occupy some similar residues of $\beta$-catenin, thereby resulting in competitive binding for $\beta$-catenin.

\section{The SHARPIN- $\beta$-catenin signaling axis is essential for tumorigenicity and invasiveness of GC cells}

Considering a key regulatory effect of SHARPIN on $\beta$-catenin stability, we investigated whether $\beta$-catenin was an important mediator of the oncogenic function of SHARPIN in GC cells. To support this notion, ectopic expression of $\beta$-catenin rescued cell proliferation, and colony formation, and anchorage-independent colony formation capacity of SHARPIN-depleted GC cells (Fig. 5a-d). Additionally, GC cell migration and invasion inhibition in response to SHARPIN knockdown was also restored by overexpression of $\beta$-catenin (Fig. 5e). More importantly, in vivo experiments demonstrated that reintroducing $\beta$-catenin also rescued tumor growth in mice subcutaneously injected with SHARPIN-depleted cells (Fig. 5f-h).

Furthermore, IHC staining of the dissected xenograft tumors confirmed the efficacies of SHARPIN knockdown and $\beta$-catenin overexpression in vivo (Fig. S4a). Consistently, the downregulation of target genes in SHARPINdepleted tumors were attenuated by ectopic expression of $\beta$-catenin (Fig. 5i). These results suggest that $\beta$-catenin
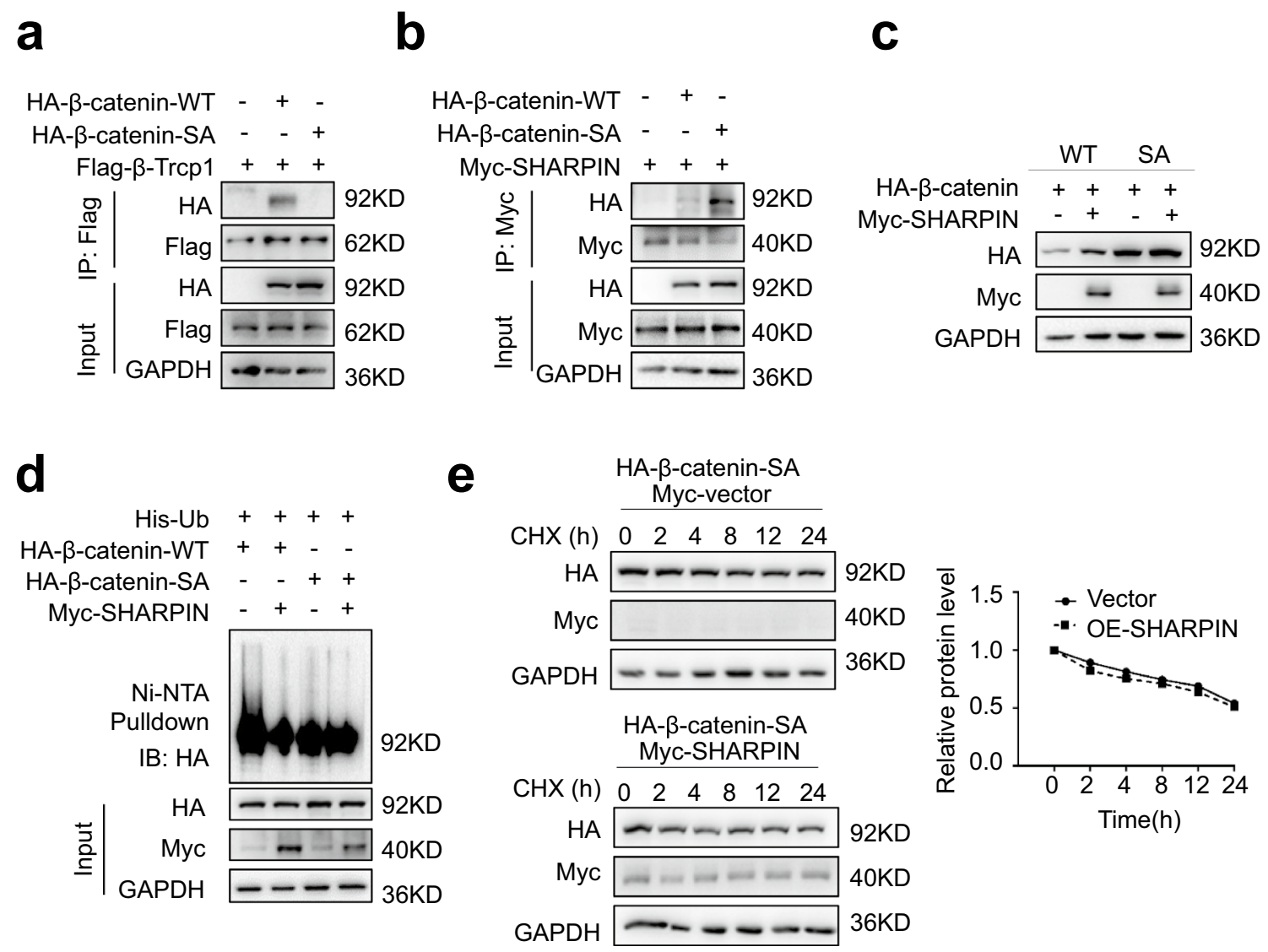

Fig. 4 SHARPIN competes with $\beta$-Trcp1 for $\beta$-catenin binding partly by occupying similar residues in $\beta$-catenin. a-b. HEK-293 T cells transfected with the indicated constructs were treated with MG132 $(20 \mu \mathrm{mol}$ for $8 \mathrm{~h})$. Then, whole cell lysates and anti-Flag or Myc immunoprecipitates were collected for immunoblot analysis with the indicated antibodies. Wild-type $\beta$-catenin is indicated as WT. SA mutant $\beta$-catenin is indicated as SA. c HEK-293 T cells transfected with $\beta$-catenin WT or SA mutation with or without Myc-SHARPIN constructs. After $48 \mathrm{~h}$, whole cell lysates were collected for immunoblot analysis. d HEK-293 T cells transfected with the indicated constructs. After $48 \mathrm{~h}$ of transfection and MG132 treatment for $8 \mathrm{~h}, \mathrm{Ni}-$ NTA pull-downs were performed for immunoblot analysis. e $293 \mathrm{~T}$ cells transfected with the $\beta$-catenin SA mutation with or without Myc-SHARPIN constructs were treated with CHX for the indicated time course. The $\beta$-catenin SA mutation level was detected by immunoblot analysis 
a

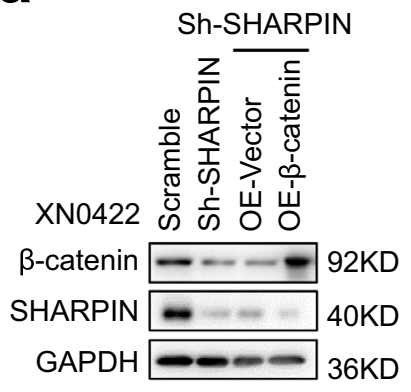

b

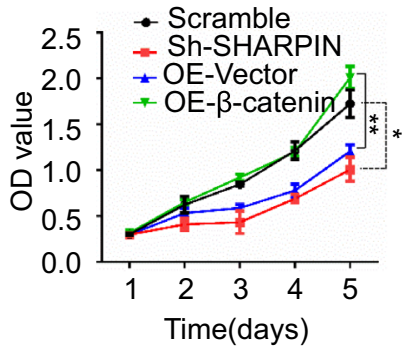

C

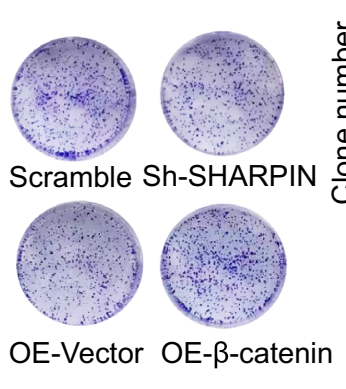

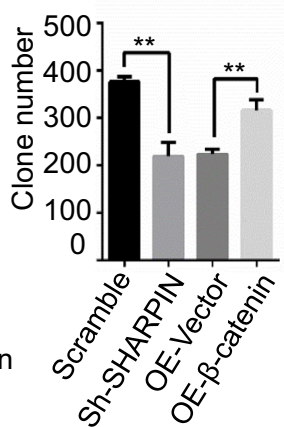

d

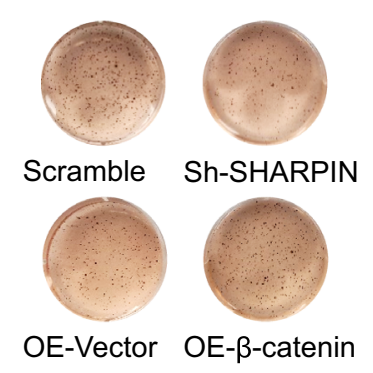

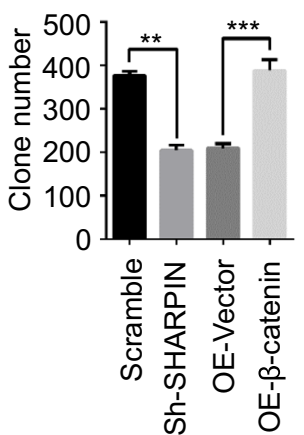

e

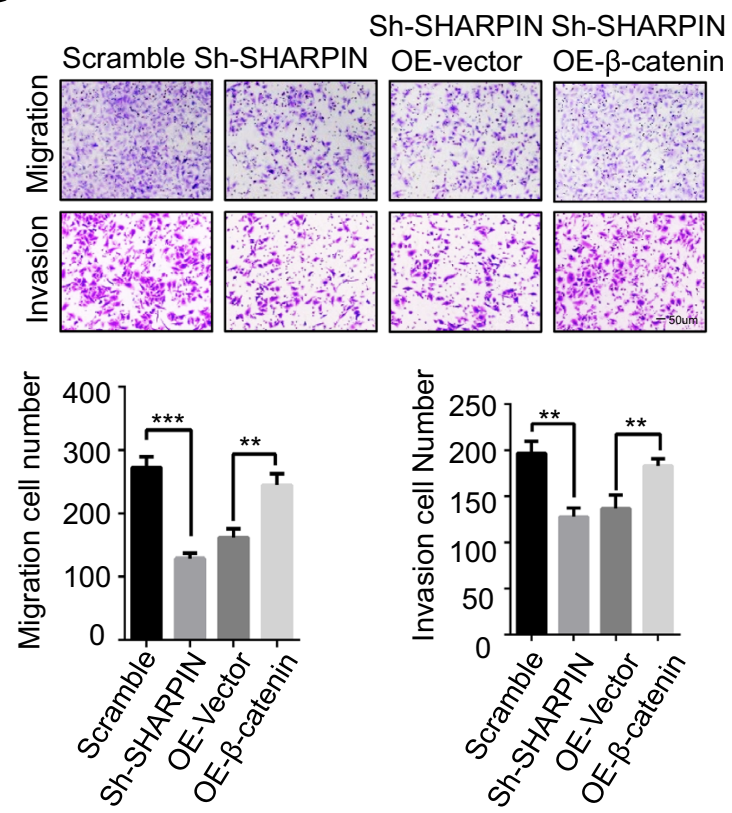

OE- $\beta$-catenin

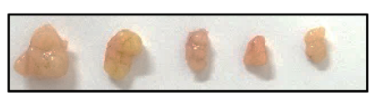

Sh-SHARPIN

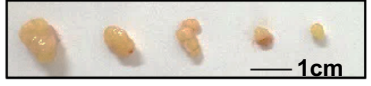

OE-vector
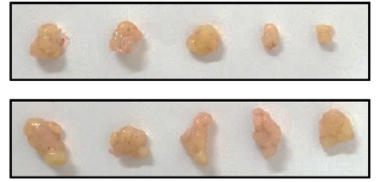

g

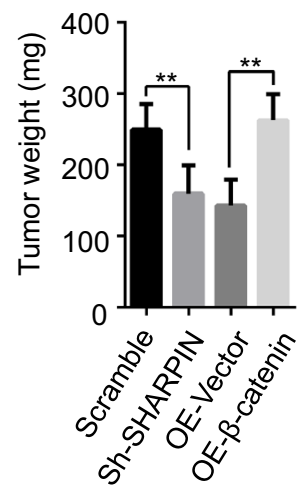

h

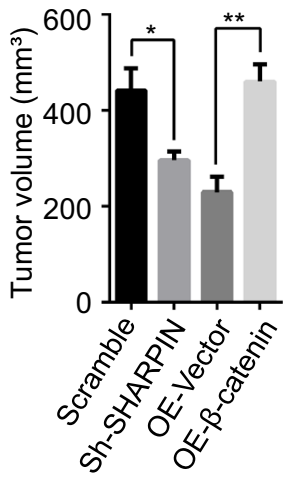

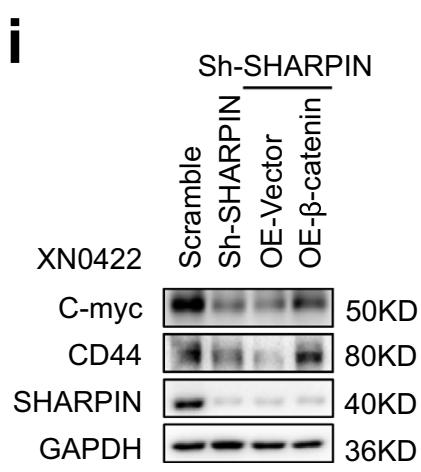


4Fig. 5 Reintroducing $\beta$-catenin in SHARPIN-depleted cells rescues their growth and tumorigenic potential. a Immunoblot analysis of SHARPIN and $\beta$-catenin in XN0422 scramble, shSHARPIN, shSHARPIN/OE-vector and shSHARPIN/OE- $\beta$-catenin cells. b-d Overexpression of $\beta$-catenin restored the proliferation and colony formation capacity of SHARPIN-depleted GC cells. CCK-8, colony formation and soft agar colony formation assays were used to examine the effect of $\beta$-catenin overexpression on the proliferation of XN0422 cells with SHARPIN knockdown. e Overexpression of $\beta$-catenin restored the migration and invasion of SHARPIN-depleted GC cells. Transwell migration and invasion assays were performed to detect the migration and invasion ability of XN0422 cells. Quantitative data are presented as the mean \pm SD. $* * * P<0.001$. f Reintroducing $\beta$-catenin restored the growth of SHARPIN-depleted GC cells. XN0422 scramble, shSHARPIN, shSHARPIN/OE-vector and shSHARPIN/OE- $\beta$ catenin cells were subcutaneously injected into the hind limbs of nude mice ( $n=5 /$ group, $1 \times 10^{6}$ cells per injection). After 5 weeks, the tumors were removed and photographed. $\mathbf{g}-\mathbf{h}$. After the tumors were dissected, the tumor weight and volume were measured $(P<0.05)$; data are presented as the mean \pm SD. $* * P<0.01$. i Immunoblot analysis of C-myc and CD44 in the dissected xenograft tumors

activity is essential for SHARPIN to maintain malignant phenotypes of GC cells.

\section{SHARPIN expression is correlated with activation of $\beta$-catenin signaling in cancer tissues and associated with disease progression of the GC patients}

We next assessed whether SHARPIN-mediated regulation of $\beta$-catenin stability is relevant to human gastric tumorigenesis. IHC results showed that 67 (37.2\%) were high expression of SHARPIN and 113 (62.8\%) were low expression in GC tissues. In corresponding adjacent normal tissues, only $36(20.0 \%)$ showed SHARPIN high expression and $144(80.0 \%)$ showed SHARPIN low expression. The expression level of SHARPIN was higher in GC tissues than in matched normal gastric mucosa $(p=0.0004)$ (Fig. 6a-c). Interestingly, SHARPIN-positive cancer cells were readily detectable at the invasive margins of tumor tissues, especially in the muscular layer and serosa (Fig. 6a), suggesting SHARPIN expression might be associated with disease progression. In deed, elevated expression of SHARPIN was correlated with higher histological grade $(p=0.032)$ and TNM stage $(p=0.033$, Table 1$)$, but not with Lauren classification, tumor size and sampling sites.

To further indicate a role of SHARPIN in disease malignancy, high intensity of SHARPIN staining in GC tissue was correlated with shorter overall survival time in about one third of GC patients (HR 2.06, (95\% CI 1.051-4.037), $P=0.0106$, Fig. 6d). Analyzing a publically available dataset further validated that high levels of SHARPIN expression was associated with dismal outcomes of GC patients (Fig. S5a, b). Moreover, both univariate and multivariate analyses revealed that high expression level of SHARPIN was an independent indicator for inferior prognosis of GC patients ( $p=0.002$ and $p=0.010$, respectively, Table 2$)$. Furthermore, a nice correlation between SHARPIN and nuclear $\beta$-catenin staining was observed in GC tissues (Fig. 6e, f). Taken together, these data indicated that SHARPIN expression is correlated with $\beta$-catenin in GC tissues and overexpression of SHARPIN in a subset of GC patients may contribute to tumor progression.

\section{Discussion}

The present study has uncovered that although SHARPIN is a key component of LUBAC, it functions as a regulator of $\mathrm{Wnt} / \beta$-catenin signaling, contributing to gastric tumorigenesis independent of linear ubiquitination. Here, we propose a model in which SHARPIN interacts with and stabilizes $\beta$-catenin by competing with the E3 ubiquitin ligase $\beta$-Trcp 1 for $\beta$-catenin binding, thereby promoting $\beta$-catenin signaling to fuel the development of GC (Fig. 7). Our results suggest that SHARPIN might be a potential target for a subgroup of GC driven by hyperactive Wnt/ $\beta$ catenin signaling.

Linear ubiquitination by LUBAC has been implicated in $\mathrm{NF}-\kappa \mathrm{B}$ signaling, immunity and chronic inflammation. Most of the known functions of SHARPIN, an essential component of LUBAC, are about inflammation and immunity through modulating $\mathrm{NF}-\kappa \mathrm{B}$ signaling [24, 25]. For instance, SHARPIN gene mutations induces spontaneous chronic proliferative dermatitis by abnormal activation of $\mathrm{NF}-\kappa \mathrm{B}$ [26]. Nevertheless, the regulatory roles of linear ubiquitination on other cell signaling pathways remain largely obscure. Our study represents a step forward in understanding how linear ubiquitination regulates $\mathrm{Wnt} / \beta$ catenin signaling in cancer settings. Previous studies have found that OTULIN, the linear ubiquitination-specific deubiquitinase, modulates Wnt signaling during embryonic angiogenesis and craniofacial and neural development [15, 27]. However, the underlying mechanism of linear ubiquitination regulating Wnt signaling has not been elucidated. Here, we find that SHARPIN recognizes and protected $\beta$-catenin from protesomal degradation, thereby promoting $\mathrm{Wnt} / \beta$-catenin signaling. In line with our results, SHARPIN is identified as a transcriptional coactivator for $\beta$-catenin onto the versican promoter region in a study of hepatocellular carcinoma [28]. We provide new evidences that SHARPIN-interacting $\beta$-catenin is protected from protesomal degradation, which may be an alternative approach to elevating $\beta$-catenin signaling during tumorigenesis.

SHARPIN is highly expressed in GC tissues. No significant difference in the expression of SHARPIN is observed between the intestinal and diffuse types of GC, suggesting 
a

Gastric cancer
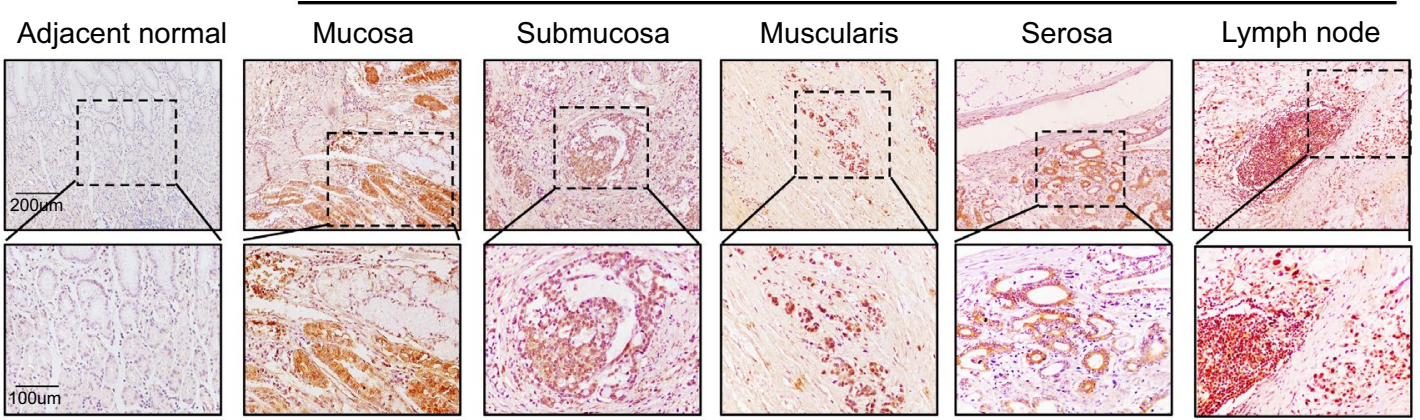

b

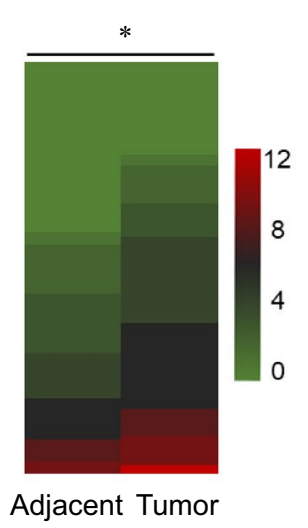

e

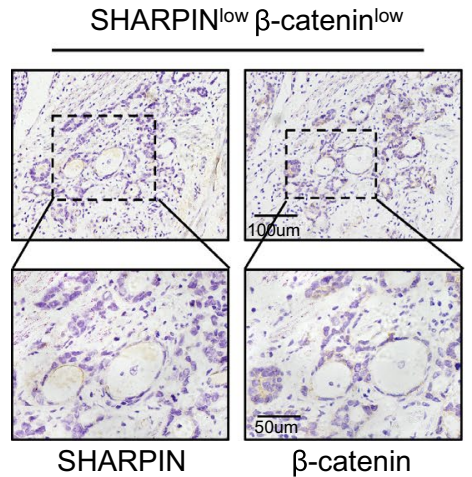

C

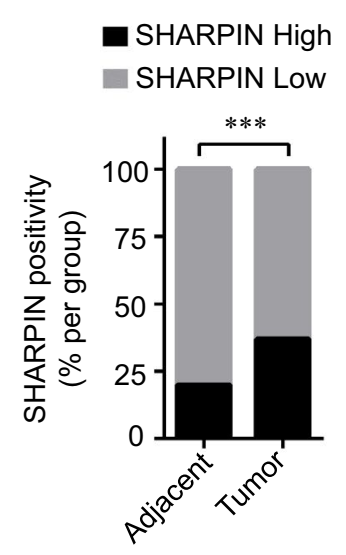

d

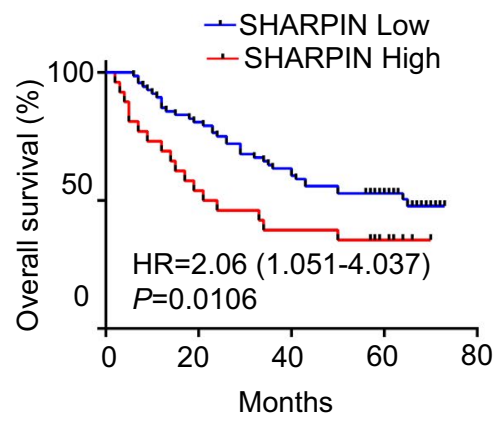

$\mathbf{f}$

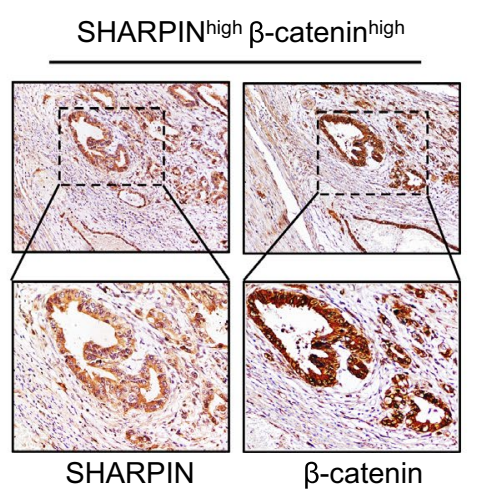

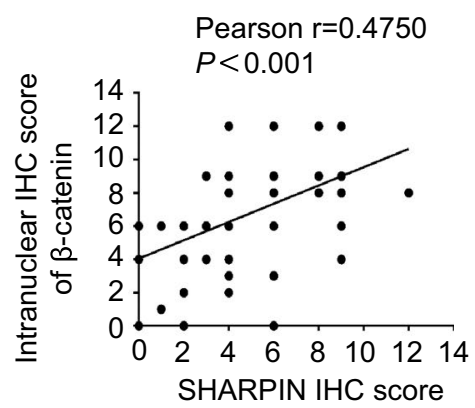

Fig. 6 High SHARPIN expression is associated with GC malignancy and correlates with $\beta$-catenin expression in GC tissue. a Representative images of SHARPIN IHC staining in GC and adjacent gastric epithelial tissues. Scale bars, $200 \mu \mathrm{m}$ and $100 \mu \mathrm{m}$. b Heat map of SHARPIN IHC scores in GC and adjacent gastric epithelial tissues. c Quantitative analysis of SHARPIN staining in GC and adjacent normal gastric epithelial tissues. Quantitative data are presented as the mean \pm SD $\left(n=180,{ }^{*} P<0.05\right)$. d Kaplan-Meier survival analysis of
GC patients. High level of SHARPIN resulted was correlated within a shorter survival time in GC patients. e-f SHARPIN and $\beta$-catenin IHC staining results in gastric cancer tissues were quantified for correlation analysis. SHARPIN was positively correlated with the expression of $\beta$-catenin in GC tissues. Representative images of both negative (case 1) and positive (case 2) SHARPIN and $\beta$-catenin IHC staining in the GC tissues. e Scale bars, $100 \mu \mathrm{m}$ and $50 \mu \mathrm{m}$ 
Table 1 Correlation between SHARPIN expression and clinicopathological characteristics of GC patients

\begin{tabular}{|c|c|c|c|c|}
\hline \multirow{3}{*}{$\begin{array}{l}\text { Characteristics } \\
\text { All patients }\end{array}$} & \multirow{3}{*}{$\begin{array}{c}\text { Cases } \\
180\end{array}$} & \multirow{2}{*}{\multicolumn{2}{|c|}{$\frac{\text { SHARPIN expression }}{\text { Low (\%) High (\%) }}$}} & \multirow[t]{3}{*}{$P$-value } \\
\hline & & & & \\
\hline & & $113(62.8 \%)$ & $67(37.2 \%)$ & \\
\hline \multicolumn{5}{|l|}{ Gender } \\
\hline Male & 128 & $77(68.14 \%)$ & $51(76.12 \%)$ & \multirow[t]{2}{*}{0.254} \\
\hline Female & 52 & $36(31.86 \%)$ & $16(23.88 \%)$ & \\
\hline \multicolumn{5}{|l|}{ Age(years) } \\
\hline$<60$ & 104 & $65(57.52 \%)$ & $39(58.21 \%)$ & \multirow[t]{2}{*}{0.928} \\
\hline$\geq 60$ & 76 & $48(42.48 \%)$ & $28(41.79 \%)$ & \\
\hline \multicolumn{5}{|l|}{ Histological grade } \\
\hline High (I+ II) & 72 & $52(46.02 \%)$ & $20(29.85 \%)$ & \multirow[t]{2}{*}{0.032} \\
\hline Low (III) & 108 & $61(53.98 \%)$ & $47(70.15 \%)$ & \\
\hline \multicolumn{5}{|l|}{ Lauren classification } \\
\hline Intestinal & 125 & $73(64.60 \%)$ & $52(77.61 \%)$ & \multirow[t]{2}{*}{0.067} \\
\hline Diffuse & 55 & $40(35.40 \%)$ & $15(22.39 \%)$ & \\
\hline \multicolumn{5}{|l|}{ T stage } \\
\hline $1+2$ & 45 & $33(29.20 \%)$ & $12(17.91 \%)$ & \multirow[t]{2}{*}{0.091} \\
\hline $3+4$ & 135 & $80(70.80 \%)$ & $55(82.09 \%)$ & \\
\hline \multicolumn{5}{|l|}{ Lymphatic invasion } \\
\hline No (N0) & 75 & $49(43.36 \%)$ & $26(38.81 \%)$ & \multirow[t]{2}{*}{0.549} \\
\hline Yes (N1) & 105 & $64(56.64 \%)$ & $41(61.19 \%)$ & \\
\hline \multicolumn{5}{|l|}{ Tumor size $(\mathrm{cm})$} \\
\hline$<5$ & 122 & $82(72.57 \%)$ & $40(59.70 \%)$ & \multirow[t]{2}{*}{0.074} \\
\hline$\geq 5$ & 58 & $31(24.43 \%)$ & $27(40.30 \%)$ & \\
\hline \multicolumn{5}{|l|}{ TNM stage } \\
\hline $\mathrm{I}+\mathrm{II}$ & 83 & $59(52.21 \%)$ & $24(35.82 \%)$ & \multirow[t]{2}{*}{0.033} \\
\hline $\mathrm{IIII}+\mathrm{V}$ & 97 & $54(47.79 \%)$ & $43(64.18 \%)$ & \\
\hline \multicolumn{5}{|l|}{ Proximal vs distal ${ }^{*}$} \\
\hline Proximal stomach & 35 & $21(27.27 \%)$ & $14(34.15 \%)$ & \multirow[t]{2}{*}{0.436} \\
\hline Distal stomach & 83 & $56(72.73 \%)$ & $27(65.85 \%)$ & \\
\hline
\end{tabular}

*Clinical information from 118 patients was available for the analysis of sampling sites

that SHARPIN overexpression is a common feature of two histological types. During gastric carcinogenesis, $\mathrm{Wnt} / \beta$-catenin signaling pathway plays crucial roles in inducing epithelial-mesenchymal transition (EMT) to promote invasiveness of cancer cells. Here we have identified SHARPIN as a novel adaptor protein to stabilize $\beta$-catenin and activate downstream signaling. Moreover, SHARPIN is required for motility and invasion of gastric cancer cells via up-regulating $\beta$-catenin protein levels. These two lines of evidence suggest that SHARPIN may be acting on $\beta$-catenin signaling to regulate EMT of gastric cancer cells. Consistently, high expression of SHARPIN predicts poor prognosis of GC patients. Since SHARPIN is overexpressed in multiples types of cancers [28-30], where $\mathrm{Wnt} / \beta$-catenin signaling pathway is frequently hyper-activated, it s potentially exploitable to block the SHARPIN- $\beta$-catenin interaction for therapeutic applications in these settings.

We have also revealed that SHARPIN could functions in a linear ubiquitination-independent manner to suppress the degradative ubiquitination modification of its interacting proteins. In addition to our report, other biological functions of SHARPIN have also been identified. For example, SHARPIN is a negative regulator of PTEN to promote tumorigenesis [31], possibly through facilitating PTEN K63linked polyubiquitination to inhibit its phosphatase activity [32]. SHARPIN is also an endogenous inhibitor of $\beta$-integrin in human prostate cancer and regulates cell adhesion and migration [33]. Consistently, we have found that SHARPIN maintains migration and invasion capacity of GC cells, albeit via stabilizing $\beta$-catenin to maintain its signaling activity. Additionally, a recent study on the SHARPIN interactome found that SHARPIN was also involved in lamellipodium formation through interaction with the Arp2/3 complex and promoted cell migration [34]. Thus, SHARPIN is a multifunctional protein that plays diverse biological roles in a context-dependent manner.

To suppress ubiquitination and degradation of $\beta$-catenin, SHARPIN functions as an adaptor protein that recognizes $\beta$-catenin, competing with the E3 ligase $\beta$-Trcp1 for binding to $\beta$-catenin. When SHARPIN is highly expressed in GC cells, $\beta$-Trcp1 fails to interact with $\beta$-catenin to mediate ubiquitination and protesomal degradation. Thus, the expression of SHARPIN is positively associated with $\beta$-catenin staining intensity and nuclear localization in a subgroup of GC tissues, which supports a potential role of the SHARPIN$\beta$-catenin axis in cancer progression. Additionally, through point mutation experiments, we found that SHARPIN and $\beta$-Trcp1 bound to similar residues in $\beta$-catenin in a mutually
Table 2 Univariate and multivariate analyses of the contribution of SHARPIN on the survival of GC patients

\begin{tabular}{|c|c|c|c|c|}
\hline & \multicolumn{2}{|l|}{ Univariate analysis } & \multicolumn{2}{|l|}{ Multivariate analysis } \\
\hline & Hazard ratio $(95 \% \mathrm{CI})$ & $p$ value & Hazard ratio $(95 \% \mathrm{CI})$ & $p$ value \\
\hline Age $(>60)$ & $0.727(0.476-1.110)$ & 0.140 & $0.747(0.486-1.148)$ & 0.183 \\
\hline Sex (male) & $1.321(0.864-2.019)$ & 0.199 & $1.118(0.721-1.734)$ & 0.619 \\
\hline Histological Grade & $1.023(0.661-1.582)$ & 0.920 & $0.973(0.621-1.525)$ & 0.904 \\
\hline TNM & $1.829(1.180-2.835)$ & 0.007 & $1.695(1.089-2.639)$ & 0.019 \\
\hline SHARPIN (high) & $1.940(1.272-2.959)$ & 0.002 & $1.770(1.143-2.741)$ & 0.010 \\
\hline
\end{tabular}




\section{High level of SHARPIN}

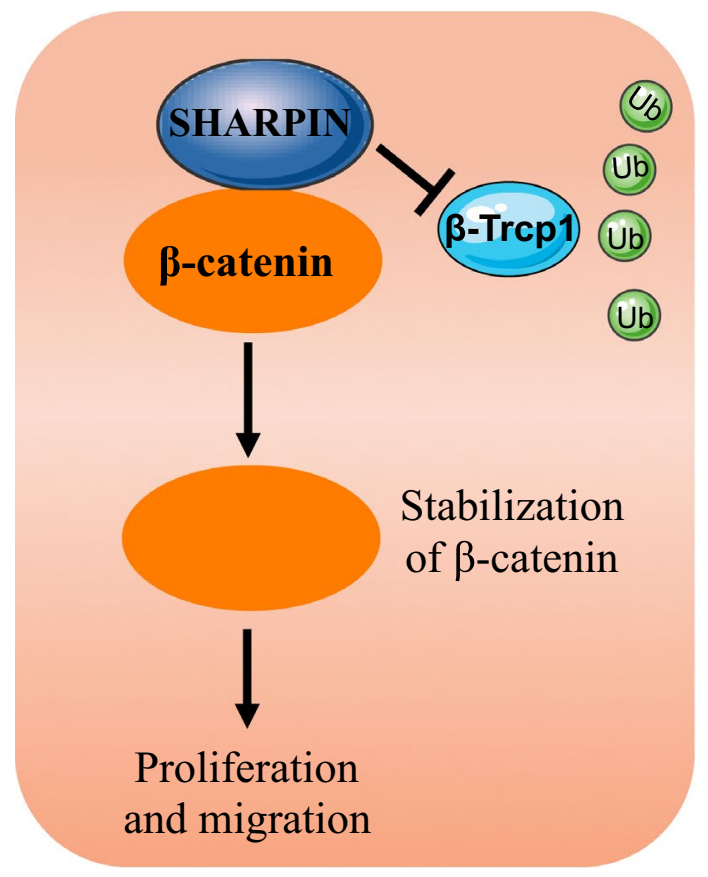

\section{Low level of SHARPIN}

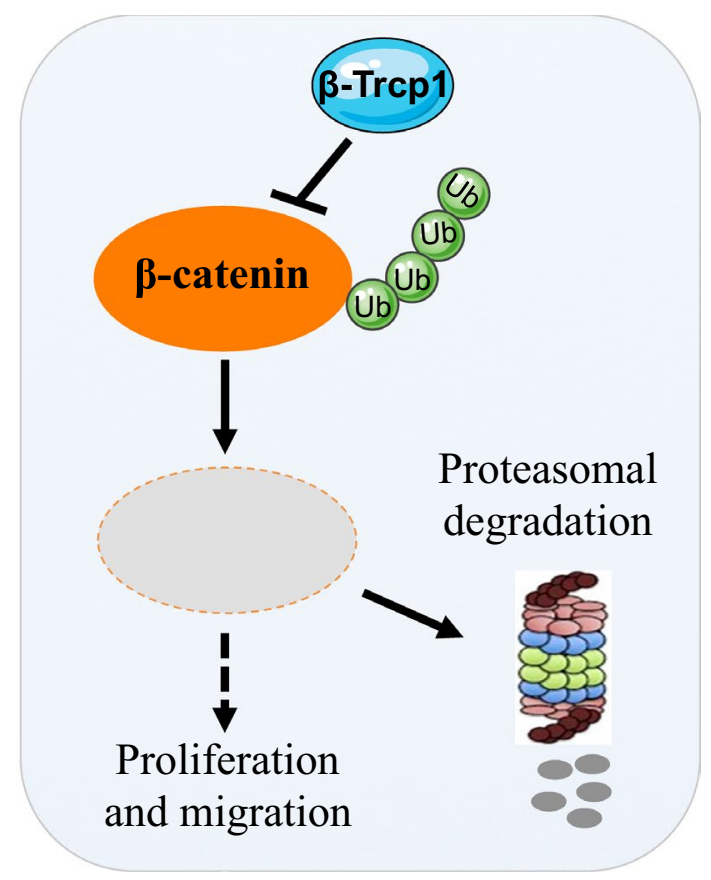

Fig. 7 Schematic model illustrating that SHARPIN stabilizes $\beta$-catenin via inhibiting degradative ubiquitination mediated by the E3 ubiquitin ligase $\beta$-Trcp 1 , thereby promoting $\beta$-catenin signaling and malignancy in a subset of human gastric cancer

exclusive manner, which resulted in attenuated ubiquitination and proteasomal degradation of $\beta$-catenin. However, further details of these mechanisms still need to be investigated. It is also possible that SHARPIN interaction with $\beta$-catenin may cause a spatial conformation of steric hindrance, thereby inhibiting the binding of $\mathrm{E} 3$ ubiquitin ligases (such as $\beta$-Trcp1 or other ligases) to $\beta$-catenin. Therefore, further studies are warranted to explore additional regulatory mechanisms in a separate study.

Together, this study revealed a previously unappreciated molecular pathway for activation of $\mathrm{Wnt} / \beta$-catenin signaling by the linear ubiquitination machinery. Blocking the SHARPIN- $\beta$-catenin interacting surface could be an effective treatment strategy against human cancers harboring deregulated $\beta$-catenin signaling.

Acknowledgements We thank Dr. Koraljka Husnjak (Institute of Biochemistry II, Goethe University Frankfurt-Medical Faculty, Frankfurt, Germany) for providing INT-Ub plasmids.

Funding This work was sponsored by the grants from the National Natural Science Foundation of China (NSFC Nos. 81822032, 91959111, and 81872027 to Bin Wang, 81672463 and 81972305 to Dongfeng Chen), Natural Science Foundation of Chongqing (No. CSTC2019JCYJJQX0027 to Bin Wang), and Founding from the Army Medical University (Nos. 2019CXLCA001, 2018XLC2023 and 2019XQY19 to Bin Wang, 2019XLC1012 to Dongfeng Chen).

\section{Compliance with ethical standards}

Conflict of interest The authors have declared no potential conflicts of interest.

\section{References}

1. Pal A, Young MA, Donato NJ. Emerging potential of therapeutic targeting of ubiquitin-specific proteases in the treatment of cancer. Cancer Res. 2014;74:4955-66.

2. Song L, Luo ZQ. Post-translational regulation of ubiquitin signaling. J Cell Biol. 2019;218:1776-86.

3. Swatek KN, Komander D. Ubiquitin modifications. Cell Res. 2016;26:399-422.

4. Bernassola F, Chillemi G, Melino G. HECT-type E3 ubiquitin ligases in cancer. Trends Biochem Sci. 2019;44:1057-75.

5. Fujita Y, Tinoco R, Li Y, Senft D, Ronai ZA. Ubiquitin ligases in cancer immunotherapy-balancing antitumor and autoimmunity. Trends Mol Med. 2019;25:428-43.

6. Kubbutat MH, Jones SN, Vousden KH. Regulation of p53 stability by Mdm2. Nature. 1997;387:299-303.

7. Grice GL, Nathan JA. The recognition of ubiquitinated proteins by the proteasome. Cell Mol Life Sci. 2016;73:3497-506.

8. Kulathu Y, Komander D. Atypical ubiquitylation-the unexplored world of polyubiquitin beyond Lys48 and Lys63 linkages. Nat Rev Mol Cell Biol. 2012;13:508-23.

9. Israël A. NF-kappaB activation: Nondegradative ubiquitination implicates NEMO. Trends Immunol. 2006;27:395-7. 
10. Sasaki K, Iwai K. Roles of linear ubiquitinylation, a crucial regulator of NF- $\mathrm{KB}$ and cell death, in the immune system. Immunol Rev. 2015;266:175-89.

11. Yang Y, Schmitz R, Mitala J, Whiting A, Xiao W, Ceribelli M, et al. Essential role of the linear ubiquitin chain assembly complex in lymphoma revealed by rare germline polymorphisms. Cancer Discov. 2014;4:480-93.

12. Ruiz EJ, Diefenbacher ME, Nelson JK, Sancho R, Pucci F, Chakraborty A, et al. LUBAC determines chemotherapy resistance in squamous cell lung cancer. J Exp Med. 2019;216:450-65.

13. Deng YZ, Yao F, Li JJ, Mao ZF, Hu PT, Long LY, et al. RACK1 suppresses gastric tumorigenesis by stabilizing the $\beta$-catenin destruction complex. Gastroenterology. 2012;142(812-23):e15.

14. Wang T, Wu H, Liu S, Lei Z, Qin Z, Wen L, et al. SMYD3 controls a Wnt-responsive epigenetic switch for ASCL2 activation and cancer stem cell maintenance. Cancer Lett. 2018;430:11-24.

15. Rivkin E, Almeida SM, Ceccarelli DF, Juang YC, MacLean TA, Srikumar $\mathrm{T}$, et al. The linear ubiquitin-specific deubiquitinase gumby regulates angiogenesis. Nature. 2013;498:318-24.

16. Wang B, Liu J, Ma LN, Xiao HL, Wang YZ, Li Y, et al. Chimeric 5/35 adenovirus-mediated Dickkopf-1 overexpression suppressed tumorigenicity of CD44 (+) gastric cancer cells via attenuating Wnt signaling. J Gastroenterol. 2013;48:798-808.

17. Kliza K, Taumer C, Pinzuti I, Franz-Wachtel M, Kunzelmann S, Stieglitz B, et al. Internally tagged ubiquitin: a tool to identify linear polyubiquitin-modified proteins by mass spectrometry. Nat Methods. 2017;14:504-12.

18. Qin ZY, Wang T, Su S, Shen LT, Zhu GX, Liu Q, et al. BRD4 promotes gastric cancer progression and metastasis through acetylation-dependent stabilization of snail. Cancer Res. 2019;79:4869-81.

19. Wang B, Jie Z, Joo D, Ordureau A, Liu P, Gan W, et al. TRAF2 and OTUD7B govern a ubiquitin-dependent switch that regulates mTORC2 signalling. Nature. 2017;545:365-9.

20. Tang Z, Li C, Kang B, Gao G, Li C, Zhang Z, et al. GEPIA: a web server for cancer and normal gene expression profiling and interactive analyses. Nucleic Acids Res. 2017;45:w98-102.

21. Yang H, Yu S, Wang W, Li X, Hou Y, Liu Z, et al. SHARPIN Facilitates p53 Degradation in Breast Cancer Cells. Neoplasia. 2017;19:84-92.

22. Nusse R, Clevers H. Wnt $/ \beta$-catenin signaling, disease, and emerging therapeutic modalities. Cell. 2017;169:985-99.

23. Liu C, Kato Y, Zhang Z, Do VM, Yankner BA, He X, et al. beta-Trcp couples beta-catenin phosphorylation-degradation and regulates Xenopus axis formation. Proc Natl Acad Sci. 1999;96:6273-8.

24. Tokunaga F, Nakagawa T, Nakahara M, Saeki Y, Taniguchi M, Sakata S, et al. SHARPIN is a component of the NF-кB-activating linear ubiquitin chain assembly complex. Nature. 2011;471:633-6.

25. Ikeda F, Deribe YL, Skånland SS, Stieglitz B, Grabbe C, FranzWachtel M, et al. SHARPIN forms a linear ubiquitin ligase complex regulating NF- $\mathrm{\kappa B}$ activity and apoptosis. Nature. 2011;471:637-41.

26. Sharma BR, Karki R, Lee E, Zhu Q, Gurung P, Kanneganti TD, et al. Innate immune adaptor MyD88 deficiency prevents skin inflammation in SHARPIN-deficient mice. Cell Death Differ. 2019;26:741-50.

27. Keusekotten K, Elliott PR, Glockner L, Fiil BK, Damgaard $\mathrm{RB}$, Kulathu Y, et al. OTULIN antagonizes LUBAC signaling by specifically hydrolyzing Met1-linked polyubiquitin. Cell. 2013; 153:1312-26.

28. Tanaka Y, Tateishi K, Nakatsuka T, Kudo Y, Takahashi R, Miyabayashi K, et al. Sharpin promotes hepatocellular carcinoma progression via transactivation of Versican expression. Oncogenesis. 2016;5:e277.

29. Zhang Y, Huang H, Zhou H, Du T, Zeng L, Cao Y, et al. Activation of nuclear factor $\kappa \mathrm{B}$ pathway and downstream targets survivin and livin by SHARPIN contributes to the progression and metastasis of prostate cancer. Cancer. 2014;120:3208-18.

30. De Melo J, Tang D. Elevation of SIPL1 (SHARPIN) Increases Breast Cancer Risk. PLoS ONE. 2015;10:e0127546.

31. He L, Ingram A, Rybak AP, Tang D. Shank-interacting proteinlike 1 promotes tumorigenesis via PTEN inhibition in human tumor cells. J Clin Invest. 2010;120:2094-108.

32. De Melo J, Lin X, He L, Wei F, Major P, Tang D, et al. SIPL1facilitated PTEN ubiquitination contributes to its association with PTEN. Cell Signal. 2014;26:2749-56.

33. Gao J, Bao Y, Ge S, Sun P, Sun J, Liu J, et al. SHARPIN is an endogenous inhibitor of $\beta 1$-integrin activation. Nat Cell Biol. 2011;13:1315-24.

34. Khan MH, Salomaa SI, Jacquemet G, Butt U, Miihkinen M, Deguchi T, et al. The Sharpin interactome reveals a role for Sharpin in lamellipodium formation via the Arp2/3 complex. J Cell Sci. 2017;130:3094-107.

Publisher's Note Springer Nature remains neutral with regard to jurisdictional claims in published maps and institutional affiliations.

\section{Affiliations}

\section{Liang Zhang ${ }^{1}$ - Qin Liu ${ }^{1} \cdot$ Ke-wei Liu ${ }^{1}$ - Zhong-yi Qin ${ }^{1}$ - Guang-xi Zhu ${ }^{1} \cdot$ Li-ting Shen ${ }^{1}$ - Ni Zhang ${ }^{1}$ - Bi-ying Liu ${ }^{1}$. Lin-rong Che ${ }^{1}$. Jin-yang $\mathrm{Li}^{1} \cdot$ Tao Wang $^{1} \cdot$ Liang-zhi Wen $^{1} \cdot$ Kai-jun Liu ${ }^{1} \cdot$ Yan Guo ${ }^{1} \cdot$ Xin-ru Yin ${ }^{1} \cdot$ Xing-wei Wang ${ }^{1}$. Zhi-hua Zhou ${ }^{2} \cdot$ Hua-liang Xiao ${ }^{3}$. You-hong Cui ${ }^{4} \cdot$ Xiu-wu Bian $^{4} \cdot$ Chun-hui Lan ${ }^{1} \cdot$ Dongfeng Chen $^{1} \cdot$ Bin Wang $^{1,4}$}

1 Department of Gastroenterology, Daping Hospital, Army Medical University (Third Military Medical University), Chongqing 400042, People's Republic of China

2 Department of Pathology, The 904 Hospital of People Liberation Army, Wuxi, People's Republic of China

3 Department of Pathology, Daping Hospital, Army Medical University (Third Military Medical University), Chongqing 400042, People's Republic of China
4 Institute of Pathology and Southwest Cancer Center, Key Laboratory of Tumor Immunopathology of Ministry of Education of China, Southwest Hospital, Army Medical University (Third Military Medical University), Chongqing 400038, People's Republic of China 